\title{
Endometrial Carcinoma in a 20 Year Old Female: A Rare Presentation
}

\author{
Meenu Gupta ${ }^{1}$, Tarik Rasool Malik', Mohd Lateef Wani ${ }^{2 *}$ and Mohammad Maqbool Lone ${ }^{1}$
}

${ }^{1}$ Department of Radiotherapy and CVTS SKIMS, Srinagar, India

${ }^{2}$ Senior Resident, CVTS SKIMS, Srinagar, India

\begin{abstract}
Carcinoma of endometrium is among the most common female pelvic malignancies. The mean age at diagnosis is 61 years. However, $5-30 \%$ of women are aged younger than 50 years at the time of diagnosis. This tumor is rare in patients less than 30 years of age. We present a case of carcinoma of endometrium in 20 year female with review of literature.
\end{abstract}

Keywords: Carcinoma endometrium; Young female; Risk factors

\section{Introduction}

Endometrial carcinoma is predominantly a disease of postmenopausal ladies and is rarely found in young females owing to lesser duration of chronic estrogen exposure. Younger patients present with clinical challenges as far as treatment options are concerned because with standard surgical treatment there is a risk of developing severe complications which include loss of fertility and surgical menopause. Compared with older women, young women have a similar stage distribution at diagnosis, the majority of cancers presenting as stage I.

\section{Case Report}

A 20 year old unmarried female with complaints of menorrhagia of nine months duration sought gynaecological consultation in March 2008. General physical examination was normal and she was haemodynamically stable. Abdominal examination did not reveal any tenderness or lump. Baseline investigations revealed mild anemia. Chest X-ray, electrocardiogram, liver functions and kidney function were normal. Ultrasound of the abdomen documented only bulky uterus. Contrast CAT scan of the abdomen and pelvis was normal. MRI was not done as CT scan was normal. On hysteroscopic examination there was a polyp in the endometrium which was resected and histopathology of the resected polyp revealed moderately differentiated adenocarcinoma.

It was decided by the surgeon and oncologist that patient should undergo surgery. Patient underwent extra fascial hysterectomy with bilateral pelvic lymph nodal sampling through laparotomy. Histopathological examination of the operated specimen revealed adenocarcinoma of endometrium which was grade II with myometrial invasion. Ovarian transposition was performed. Patient received external beam radiation therapy as adjuvant treatment to the pelvis to a total dose of 45 Grays in 20 fractions. Presently patient is on follow up and disease free.

\section{Discussion}

Carcinoma of the endometrium is among the most common gynaecological malignancies of female genital tract and accounts for $6 \%$ of all cancers in women and $2 \%$ of cancer deaths. In the United States and ranks as $4^{\text {th }}$ most common malignancy in women after breast, bronchopulmonary and colorectal cancers [1]. Endometrial carcinoma is primarily a disease of menopausal and postmenopausal females with the peak incidence in women aged 55-65 years. Approximately $75 \%$ of patients are aged 50 years and older and only $5 \%$ are younger than 40 years. It is rare below the age of 30 years [1]. Risk factors such as obesity, nulliparity, irregular menstruation and possibly Polycystic
Ovarian Syndrome (PCOS) are relevant in the development of this cancer in this age group [2]. These patients are at an increased risk of synchronous tumors of the ovary when compared with the general population.

The diagnosis of endometrial cancer in young, premenopausal females may present the patient and the physician with question of conservative management or ovarian preservation. Standard treatment for these patients includes hysterectomy with bilateral salpingo-oophorectomy and results in absolute loss of fertility and surgical menopause with its attendant complications [3]. BSO seems to lead to better disease-free survival in young endometrial cancer patients, especially with stage I disease, but not to improved overall survival. In the absence of risk factors, a more conservative approach to surgical staging may be possible in young women with early stage low grade endometrial cancer but BSO should be strongly considered as part of the surgical treatment [4]. Several authors have described successful pregnancies or cryopreservation of embryos following fertility preserving surgery in these patients and none has reported cryopreservation of ovarian tissue so far [5].

It has been observed that the younger patients with cancer of uterus carry a higher risk for the development of ovarian malignancy in the range of 5-29\% [3]. Synchronous cancers represented less than $3 \%$ of the 56,986 epithelial ovarian cancer cases, regardless of the time interval between detection of both cancers [6]. Therefore careful preoperative and intraoperative assessment of adnexia is mandatory in this subset of patients and those patients who wish to preserve ovarian function should be counselled about the increased risk of the development of co-existing ovarian cancer. Women with synchronous ovarian and endometrial cancers exhibit favourable survival outcomes as compared with patients with single ovarian cancers, even after adjusting for demographic, prognostic (including stage), and treatment characteristics [6].

\section{References}

1. Dai FR, Peng GQ, Zhang Y, Chen CX (2005) Clinical observation of young middle-aged and elderly women with endometrial carcinoma. Zhong Nan Da Xue Xue Bao Yi Xue Ban 30: 690-693.

${ }^{*}$ Corresponding author: Mohd Lateef Wani, Senior Resident, CVTS SKIMS Srinagar, India, E-mail: latifs_dr@yahoo.com

Received April 05, 2012; Accepted May 03, 2012; Published May 15, 2012

Citation: Gupta M, Malik TR, Wani ML, Lone MM (2012) Endometrial Carcinoma in a 20 Year Old Female: A Rare Presentation. J Clin Case Rep 2:146. doi:10.4172/2165-7920.1000146

Copyright: ( 2012 Gupta M, et al. This is an open-access article distributed under the terms of the Creative Commons Attribution License, which permits unrestricted use, distribution, and reproduction in any medium, provided the original author and source are credited. 
Citation: Gupta M, Malik TR, Wani ML, Lone MM (2012) Endometrial Carcinoma in a 20 Year Old Female: A Rare Presentation. J Clin Case Rep 2:146. doi:10.4172/2165-7920.1000146

2. Soliman PT, Oh JC, Schmeler KM, Sun CC, Slomovitz BM, et al. (2005) Risk factors for young premenopausal women with endometrial cancer. Obstet Gynecol 105: 575-580.

3. Walsh C, Holschneider C, Hoang Y, Tieu K, Karlan B, et al. (2005) Coexisting ovarian malignancy in young women with endometrial cancer. Obstet Gyneco 106: 693-699.

4. Richter CE, Qian B, Martel M, Yu H, Azodi M, et al. (2009) Ovarian preservation and staging in reproductive-age endometrial cancer patients. Gynecol Onco 114: $99-104$
5. Yamazawa K, Hirai M, Fujito A, Nishi H, Terauchi F, et al. (2007) Fertilitypreserving treatment with progestin, and pathological criteria to predict responses, in young women with endometrial cancer. Hum Reprod 22: 19531958

6. Williams MG, Bandera EV, Demissie K, Rodríguez-Rodríguez L (2009) Synchronous primary ovarian and endometrial cancers: a population-based assessment of survival. Obstet Gynecol 113: 783-789. 